\title{
TITLE:
}

\section{Conserved charges in general relativity}

$\operatorname{AUTHOR}(\mathrm{S})$ :

Aoki, Sinya; Onogi, Tetsuya; Yokoyama, Shuichi

CITATION:

Aoki, Sinya ...[et al]. Conserved charges in general relativity.

International Journal of Modern Physics A 2021, 36(10): 2150098.

\section{ISSUE DATE:}

2021-04

URL:

http://hdl.handle.net/2433/266162

\section{RIGHT:}

(C) The Author(s); This is an Open Access article published by World Scientific Publishing Company. It is distributed under the terms of the Creative Commons Attribution 4.0 (CC BY) License which permits use, distribution and reproduction in any medium, provided the original work is properly cited. 


\title{
Conserved charges in general relativity
}

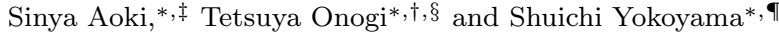 \\ * Center for Gravitational Physics, \\ Yukawa Institute for Theoretical Physics, Kyoto University, \\ Kitashirakawa Oiwake-cho, Sakyo-Ku, Kyoto 606-8502, Japan \\ $\dagger$ Department of Physics, Osaka University, \\ Toyonaka, Osaka 560-0043, Japan \\ ${ }^{\ddagger}$ saoki@yukawa.kyoto-u.ac.jp \\ §onogi@phy.sci.osaka-u.ac.jp \\ 『shuichi.yokoyama@yukawa.kyoto-u.ac.jp
}

Received 4 March 2021

Accepted 29 March 2021

Published 14 April 2021

\begin{abstract}
We present a precise definition of a conserved quantity from an arbitrary covariantly conserved current available in a general curved space-time with Killing vectors. This definition enables us to define energy and momentum for matter by the volume integral. As a result we can compute charges of Schwarzschild and BTZ black holes by the volume integration of a delta function singularity. Employing the definition we also compute the total energy of a static compact star. It contains both the gravitational mass known as the Misner-Sharp mass in the Oppenheimer-Volkoff equation and the gravitational binding energy. We show that the gravitational binding energy has the negative contribution at maximum by $68 \%$ of the gravitational mass in the case of a constant density. We finally comment on a definition of generators associated with a vector field on a general curved manifold.
\end{abstract}

Keywords: Field theory on curved space-time; conserved charge; black hole; general relativity; energy-momentum tensor; compact star; Oppenheimer-Volkoff equation.

PACS numbers: 11.30.Cp, 11.30.Fs, 11.90.+t

\section{Introduction}

Since Einstein submitted papers on general relativity, $\frac{1}{,}$ classical or quantum field theory on a curved space-time has extensively been investigated. When space-time

$\ddagger$ Corresponding author.

This is an Open Access article published by World Scientific Publishing Company. It is distributed under the terms of the Creative Commons Attribution 4.0 (CC BY) License which permits use, distribution and reproduction in any medium, provided the original work is properly cited. 
is curved, the physical quantities defined on flat space-time are required to be modified suitably in accordance with general covariance. For example, a conserved current, which exists in the presence of global symmetry in the system,, 2 is modified to be a covariantly conserved one on a general curved space-time.

However there has been no general argument to define a conserved charge from a covariantly conserved current, which inevitably causes a problem to define energy and momentum. Einstein originally argued that the conservation law of energy and momentum for matter follows as long as they are combined with those for gravitational field ${ }^{1}$ (see also Refs. 3-5). The corresponding energy-momentum tensor for the gravitational field, however, is not covariant under general coordinate transformation. As a result the energy defined as in the case of flat space-time depends on a coordinate system and conserves only in the particular frame.

One way to circumvent this issue is to define energy locally on the asymptotic region of space-time called quasi-local energy. Initially the quasi-local energy and momentum were studied on an asymptotically flat space-time by recasting gravity system into the Hamiltonian dynamics known as the ADM formalism ${ }^{6,7}$ (see also Ref. 8). They are defined by a surface integral in the asymptotic region, by which the invariance under a class of general coordinate transformations preserving a boundary condition was achieved. This result has been further extended for a more general curved space-time with surface terms suitably incorporated..$^{-} \underline{-13}$ A caveat in this extension is that boundary terms accompany with divergence even in the flat space-time, so that one needs to subtract it by comparing a reference frame or by adding local counterterms.

The authors of this paper investigated a property of a black hole holographically realized by the flow equation method. ${ }^{14}$ In this study, we encountered a situation to evaluate the energy of the total system with matter spread all over the space, which is required to be evaluated by the volume integral of the energy density. We reached a manifestly covariant definition of a conserved charge from a covariantly conserved current in a general curved space-time, which extends the one in Ref. 15, and improves the one given in Refs. 16 and 17 for special backgrounds. This allows us to define energy and momentum for matter in a form of the volume integral at an arbitrary time slice of a given curved space-time. A virtue is to enable one to evaluate charges of black holes just like an electric charge of the electron in electromagnetism by the volume integral of an integrable singularity such as the delta function. Furthermore, applying our definition to the energy of a compact star, we discover a correction to the mass formula obtained from the Oppenheimer-Volkoff equation, which represents a contribution from the gravitational interaction and becomes $68 \%$ of the mass at the maximum for a constant density.

\section{Conserved Charge from Covariantly Conserved Current}

Consider any classical or quantum field theory on a general curved space-time. Suppose there exists a covariantly conserved current $J^{\mu}, \nabla_{\mu} J^{\mu}=0$, where $\nabla_{\mu}$ 
is the covariant derivative for the metric $g_{\mu \nu}$. Then we claim that the following quantity is conserved under the given time evolution

$$
Q(t):=\int_{M_{t}} d^{d-1} \mathbf{x} \sqrt{|g|} J^{0}(t, \mathbf{x})
$$

where $M_{t}$ represents a time slice of the space-time $M$ at the time $t, g$ denotes the determinant of $g_{\mu \nu}$, and $d$ is the dimension of the space-time $M$. If there exists boundary for $M_{t}$, we set the boundary condition for the fields to fall off sufficiently fast at boundary of $M_{t}$ for all $t$. We emphasize that $g$ is the determinant of the metric in the total space-time, which contains the time components.

To show this, we assume the space-time has the foliation structure for simplicity. (The same argument is used in literature, for example, see Ref. 18.) Let us consider the same quantity defined by (1) at another time slice with $t^{\prime}$ greater than $t$, and take a submanifold $M^{\prime}$ with the foliation structure whose boundary contains $M_{t}$ and $M_{t^{\prime}}$. Such a manifold may be written formally as $M^{\prime}=\prod_{t \leq s \leq t^{\prime}} M_{s}$. Under the boundary condition, the difference between $Q\left(t^{\prime}\right)$ and $Q(t)$ becomes

$$
Q\left(t^{\prime}\right)-Q(t)=\int_{M^{\prime}} d^{d} x \partial_{\mu}\left(\sqrt{|g|} J^{\mu}(t, \mathbf{x})\right)=0,
$$

where we used $\partial_{\mu}\left(\sqrt{|g|} J^{\mu}(t, \mathbf{x})\right)=\sqrt{|g|} \nabla_{\mu} J^{\mu}(t, \mathbf{x})=0$. This proves that $Q(t)$ is independent of $t$. The charge $Q$ is a scalar under the assumption, though the generalization is straightforward. Note that $Q$ is not a scalar if it is defined from a higher rank tensor rather than the vector.

This formula can be applied to the computation of a conserved charge for any gravitational systems with a Killing vector. A covariantly conserved current associated with a Killing vector $\xi$ can be constructed as

$$
J^{\mu}=T_{\nu}^{\mu} \xi^{\nu},
$$

where $T^{\mu}{ }_{\nu}$ is the given energy-momentum tensor for matter. It can be easily shown that this is covariantly conserved by using $\nabla_{\mu} T^{\mu}{ }_{\nu}=0$ and $\nabla_{\mu} \xi_{\nu}+\nabla_{\nu} \xi_{\mu}=0$. Note that the definition of a conserved charge using Eq. (3) in $d=4$ appears in Refs. 15, 19 and 20, but the formula has been rarely used in literature as far as we know. If $\xi^{\mu}$ is a Killing vector associated with the time translation, the conserved charge becomes the total energy of the system

$$
E=\int_{M_{t}} d^{d-1} \mathbf{x} \sqrt{|g|} T_{\mu}^{0} \xi^{\mu}
$$

which agrees with the standard definition of the energy in the flat background with $\xi^{\mu}=-\delta_{0}^{\mu}$. In Sec. 3, we compute charges of several black holes by using this formula.

\section{Conserved Charges of Black Holes}

In this section we compute a conserved charge for Schwarzschild and BTZ black holes employing the presented formula. 


\section{S. Aoki, T. Onogi \&s S. Yokoyama}

\subsection{Schwarzschild black hole}

In order to explain the key idea of the calculation we start with the simplest setup. That is, we begin with the Einstein equation

$$
R_{\mu \nu}-\frac{1}{2} g_{\mu \nu} R+\Lambda g_{\mu \nu}=0
$$

This is satisfied by the Schwarzschild black hole solution:

$$
d s^{2}=-f(r)\left(d x^{0}\right)^{2}+\frac{1}{f(r)} d r^{2}+r^{2} \tilde{g}_{i j} d x^{i} d x^{j}
$$

where $r$ is the radial coordinate and the $(d-2)$-dimensional manifold fibered over the cone is an Einstein manifold, whose Ricci tensor is given by ${ }^{(d-2)} R_{i j}=(d-3) k \tilde{g}_{i j}$ with a constant $k$, and

$$
f(r)=\frac{-2 \Lambda r^{2}}{(d-2)(d-1)}+k-\frac{2 G_{N} M}{r^{d-3}} .
$$

Note that for a positive or nonpositive $k$ the submanifold is compact or noncompact, respectively.

Since this is a static solution, there exists a Killing vector with $\xi^{\mu}=-\delta_{0}^{\mu}$, which corresponds to the time translation. Thus the corresponding charge is the energy of the system:

$$
E=\int d^{d-1} \mathbf{x} \sqrt{|g|}\left(-T_{0}^{0}\right)
$$

where the matter energy-momentum tensor is given by

$$
T_{\mu \nu}=\frac{1}{8 \pi G_{N}}\left(R_{\mu \nu}-\frac{1}{2} g_{\mu \nu} R+\Lambda g_{\mu \nu}\right),
$$

with $G_{N}$ the Newton constant. According to the equation of motion (5) this energymomentum tensor seems to vanish on shell, but it does not. We emphasize that it vanishes except a singularity located at $r=0$. This singularity contributes to the charge.

In order to evaluate the contribution, we first compute the energy by expanding the energy-momentum tensor in terms of the weak field around $r=\infty$. That is, we write $f=\bar{f}+\delta f$ with $\delta f=-2 G_{N} M / r^{d-3}$ and expand the stress tensor perturbatively around $\bar{f}$ to extract a pole. This can be done by separating the metric into the regular part $\bar{g}_{\mu \nu}$ and the singular part $h_{\mu \nu}$, the latter of which is given by

$$
h_{\mu \nu} d x^{\mu} d x^{\nu}=-\delta f\left(d x^{0}\right)^{2}+\left(\frac{1}{f}-\frac{1}{\bar{f}}\right) d r^{2} .
$$


At the leading order, we have

$$
\begin{aligned}
T_{0}^{0}= & -\frac{1}{2}\left(\frac{1}{\sqrt{|\bar{g}|}} \partial_{\mu}\left(\sqrt{|\bar{g}|} \bar{g}^{\mu \nu} \partial_{\nu} h_{0}^{0}\right)-\bar{f}^{-1} \bar{f}^{\prime 2} h_{0}^{0}\right) \\
& +\bar{\nabla}^{0} \bar{\nabla}_{\sigma} h_{0}^{\sigma}-h_{0}^{0}-\frac{1}{2} \bar{\nabla}_{\mu} \bar{\nabla}_{\sigma} h^{\sigma \mu}+\cdots
\end{aligned}
$$

where $\bar{\nabla}_{\mu}$ is the covariant derivative with respect to the metric $\bar{g}_{\mu \nu}$, and the ellipsis represents the higher-order terms with respect to $h$. This must vanish except at the origin, and indeed this can be written as

$$
T_{0}^{0}=\frac{d-2}{16 \pi G_{N} r^{d-2}} \partial_{r}\left(r^{d-3} \delta f\right)+\cdots,
$$

which has the desired property. Plugging this into (8) we can compute the charge as

$$
E=-\int d^{d-1} \mathbf{x} \sqrt{|\tilde{g}|} \frac{d-2}{16 \pi G_{N}} \partial_{r}\left(r^{d-3} \delta f\right)=\rho V_{d-2},
$$

where $V_{d-2}=\int d^{d-2} x \sqrt{|\tilde{g}|}$ is the volume of the Einstein manifold with $\tilde{g}$ being the determinant of $\tilde{g}_{i j}$, and $\rho=(d-2) M /(8 \pi)$ is the energy or mass density. To evaluate $r$ integral we employ the Stokes' theorem. Note that the higher-order terms do not contribute to the surface integral. Our result reproduces the known result obtained by other methods (for example, see Eq. (2.5) in Ref. 11).

On the other hand, it is also possible to compute the contribution of the singularity by the direct calculation of the Ricci tensor. From the direct calculation one finds that the first term in Eq. (12) is indeed exact. More detailed results are as follows:

$$
\begin{aligned}
R_{0}^{0} & =-\frac{1}{2 r^{d-2}} \partial_{r}\left(r^{d-2} \partial_{r} f(r)\right)=R_{r}^{r}, \\
R_{j}^{i} & =\delta_{j}^{i}\left[\frac{(d-3) k}{r^{2}}-\frac{1}{r^{d-2}} \partial_{r}\left(r^{d-3} f(r)\right)\right],
\end{aligned}
$$

which leads to a form of $T^{\mu}{ }_{\nu}$ proportional to a delta function:

$$
\begin{aligned}
& T_{0}^{0}=\frac{d-2}{16 \pi G_{N} r^{d-2}} \partial_{r}\left(r^{d-3} \delta f(r) \theta(r)\right)=-\rho \frac{\delta(r)}{r^{d-2}}=T_{r}^{r}, \\
& T^{i}{ }_{j}=-\delta_{j}^{i} \frac{\rho}{d-2} \frac{r \partial_{r} \delta(r)}{r^{d-2}}=\delta_{j}^{i} \frac{\rho}{d-2} \frac{\delta(r)}{r^{d-2}} .
\end{aligned}
$$

We here inserted the step function $\theta$ with $\theta(0)=0$ for the singular term in (7) to explicitly extract the singular contribution, and used $\delta(r)=\frac{d \theta(r)}{d r}$. Writing $\delta(\bar{r})$ in terms of $\delta^{(d)}(\mathbf{x})$, this agrees with a result at $d=4$ by distributional techniques. ${ }^{22}$ Thus the matter energy-momentum tensor $T^{\mu}{ }_{\nu}$ for the Schwarzschild black hole can be understood as a distribution.

Although we do not encounter any mathematical problem to derive this result, one may wonder the physical validity to perform the volume integral of the constant 
$x^{0}$ slice, which becomes timelike inside the horizon. ${ }^{\mathrm{a}}$ To clear up this subtlety, let us consider a simplified situation where the cosmological constant vanishes with $k=1$. Then we can move to the Eddington-Finkelstein coordinates $^{24,25}$

$$
d s^{2}=-(1+u) d \tau^{2}-2 u d \tau d r+(1-u) d r^{2}+r^{2} \tilde{g}_{i j} d x^{i} d x^{j},
$$

where $u=-2 G_{N} M / r^{d-3}$ and we changed the time variable from $x^{0}$ to $\tau=x^{0}-g(r)$ with $\frac{d g(r)}{d r}=\frac{u}{1+u}$. Then the unit normal vector to the constant $\tau$ slice defined by $n_{\mu}=-\frac{1}{\sqrt{1-u}} \delta_{\mu}^{\tau}$ is always well-defined and timelike for any nonzero $r$. The conserved energy, which takes the same form due to its manifest general covariance, is computed as

$$
E=-\int_{M_{\tau}} d^{d-1} \mathbf{x} \sqrt{|g|} T_{\tau}^{\tau}=\rho V_{d-2},
$$

which agrees with Eq. (13), where $T_{\tau}^{\tau}=\frac{(d-2)}{16 \pi G_{N}} \frac{\partial_{r}\left(r^{d-3} u\right)}{r^{d-2}}$.

\subsection{Reissner-Nordström black hole}

In what follows, we perform the same computation of a mass of a charged black hole in general $d$ dimensions, for which the metric is given in the same form as (6) except that $f(r)$ is replaced by $f_{q}(r)=f(r)+\frac{d-3}{d-2} 8 \pi G_{N} q^{2} r^{-2(d-3)}$, together with the gauge potential $A_{\mu}=\left(-\frac{q}{r^{d-3}}+\frac{q}{r_{+}^{d-3}}\right) \delta_{\mu}^{0}$, where $q, r_{+}$are constants. ${ }^{21}$ This configuration of gravitational and gauge fields satisfies the equations of motion given by

$$
G_{\mu \nu}+\Lambda g_{\mu \nu}=8 \pi G_{N}\left(T_{\mu \nu}^{G}+T_{\mu \nu}^{A}\right), \quad \nabla_{\mu} F_{\nu}^{\mu}=J_{\nu},
$$

where $F_{\mu \nu}:=\nabla_{\mu} A_{\nu}-\nabla_{\nu} A_{\mu}$ and $T_{\mu \nu}^{A}:=F_{\mu}{ }^{\alpha} F_{\nu \alpha}-\frac{1}{4} g_{\mu \nu} F_{\alpha \beta} F^{\alpha \beta}$. Here, $T_{\mu \nu}^{G}$ and $J_{\nu}$ explicitly represent the singular contributions of the metric and the gauge potential at $r=0$, respectively. Explicitly $\left(T^{G}+T^{A}\right)^{0}{ }_{0}$ is given in (16) by replacing $\delta f$ with $\delta f_{q}=\delta f+\frac{d-3}{d-2} 8 \pi G_{N} q^{2} r^{-2(d-3)}$. This also agrees with the distributional result at $d=4 .^{23}$

Since this metric is also static, the energy defined by (8) is conserved. However this charge diverges, due to the contribution of the electromagnetic field. Physically, this divergence can be interpreted as that of the self-energy for the charged point particle. Indeed it remains even for the flat space-time with $M=0$ and $\Lambda=0$. Therefore, classically, the charged black hole has the infinite energy due to the infinite electromagnetic energy. Thus the renormalization as well as the quantization of the gauge field on the curved space are needed to fix this problem, as is the case on the flat space.

Fortunately, since $\nabla_{\mu}\left(T^{G}\right)^{\mu}{ }_{0}=0$ (thus $\nabla_{\mu}\left(T^{A}\right)^{\mu}{ }_{0}=0$ ), we can define an energy from the covariantly conserved $T^{G}$ alone without electromagnetic energy as

$$
\left(T^{G}\right)^{0}{ }_{0}=-\frac{(d-2)}{16 \pi G_{d} r^{d-2}} \partial_{r}\left(r^{d-3} \delta f(r)\right),
$$

\footnotetext{
${ }^{a}$ We would like to thank Profs. Sasaki, Shiromizu and Sugimoto for raising this question and for discussion using the Penrose diagram.
} 
where $\delta f$ is given before. We thus obtain

$$
E_{G}=\int d^{d-2} \mathbf{x} \int d r \sqrt{|g|}\left(T^{G}\right)^{0}{ }_{0} \xi^{0}=V_{d-2} \rho,
$$

which reproduces the result in Ref. 21 as a special case choosing a sphere as the internal manifold.

This system allows another conserved quantity, thanks to the invariance under the $U(1)$ gauge transformation by $\delta A_{\mu}=\partial_{\mu} \theta$, which leads to

$$
\partial_{\mu} j^{\mu}=0, \quad j^{\mu}=\nabla_{\nu}\left(\sqrt{|g|} F^{\mu \nu}\right)
$$

without using the Maxwell equation. According to our prescription, $Q_{c}=$ $\int d^{d-2} x \int d r \sqrt{|g|} J^{0}$ with $J^{0}=j^{0} / \sqrt{|g|}$ gives the conserved electric charge, which is evaluated as $Q_{c}=V_{d-2}(d-3) q$. At $d=4$ for $k>0, Q_{c}=4 \pi q$.

\subsection{BTZ black hole}

As a final example, we compute a charge different from a mass. To this end we consider a BTZ black hole and compute its angular momentum. ${ }^{26,27}$ The metric

$$
d s^{2}=-f(r) d t^{2}+\frac{1}{f(r)} d r^{2}+r^{2}(d \phi-\omega(r) d t)^{2},
$$

where

$$
f(r)=\frac{r^{2}}{L^{2}}-2 G_{N} M \theta(r)+\frac{G_{N}^{2} J^{2}}{4 r^{2}}, \quad \omega(r)=\frac{G_{N} J}{2 r^{2}}
$$

with $M, J$ are constants, satisfies the Einstein equation in three dimensions. We insert the step function to the constant part to emphasize that this solution is valid except the origin.

This BTZ black hole has not only a Killing vector with respect to the time translation but also the one which rotates the system, $\xi^{\mu}=\delta_{\phi}^{\mu}$. As in the previous cases the first one defines the mass, which can be similarly computed as $E=\frac{M}{4}$. On the other hand, the second Killing vector defines an angular momentum:

$$
P_{\phi}=\int d^{2} x \sqrt{|g|} T_{\phi}^{0}
$$

$T_{\phi}^{0}$ is computed from the Einstein tensor as $T^{0}{ }_{\phi}=-\frac{1}{16 \pi G_{N} r} \partial_{r}\left(r^{3} \omega^{\prime}(r)\right)$. Thus we find $P_{\phi}=\frac{J}{8}$, which reproduces the known result. ${ }^{26,27}$

\section{Mass of a Compact Star}

In this section we apply a conserved charge to the computation of the total energy of a static compact star setting $k=1$ with $d \geq 3$. 
S. Aoki, T. Onogi \& S. Yokoyama

\subsection{Oppenheimer-Volkoff equation}

Let us consider a spherically symmetric system such that the metric is given by

$$
d s^{2}=-f(r)\left(d x^{0}\right)^{2}+h(r) d r^{2}+r^{2} \tilde{g}_{i j} d x^{i} d x^{j},
$$

and the matter energy-momentum tensor is described by the perfect fluid as

$$
T_{0}^{0}=-\rho(r), \quad T_{r}^{r}=P(r), \quad T_{j}^{i}=\delta_{j}^{i} P(r),
$$

where $\rho(r)$ is the density and $P(r)$ is the pressure. From the Einstein equation, we can derive useful formulas

$$
P(r)+\rho(r)=\frac{(d-2)\left(\frac{h^{\prime}(r)}{h(r)}+\frac{f^{\prime}(r)}{f(r)}\right)}{16 \pi G_{N} r h(r)}, \quad P^{\prime}(r)=-\frac{(P(r)+\rho(r))}{2} \frac{f^{\prime}(r)}{f(r)} .
$$

From these equations we can derive the Oppenheimer-Volkoff or TOV equation ${ }^{28,29}$

$$
\begin{aligned}
\frac{d P(r)}{d r}= & -\frac{G_{N} \rho(r) M(r)}{r^{d-2}}\left(1+\frac{P(r)}{\rho(r)}\right) h(r) \\
& \times\left\{d-3+\frac{r^{d-1}}{(d-2) M(r)}\left(8 \pi P(r)-\frac{2 \Lambda}{(d-1) G_{N}}\right)\right\},
\end{aligned}
$$

where

$$
\frac{1}{h(r)}:=\frac{-2 \Lambda r^{2}}{(d-2)(d-1)}+1-\frac{2 G_{N} M(r)}{r^{d-3}}
$$

and

$$
M(r)=\frac{8 \pi}{d-2} \int_{0}^{r} d s s^{d-2} \rho(s)
$$

In order for this system to describe a static compact star we impose a boundary condition such that the pressure vanishes and the energy-momentum tensor is covariantly conserved at the surface of the star. This makes the pressure and the density vanish outside the star, so that the metric becomes the Schwarzschild outside the star, namely $f(r)=1 / h(r)$ with $M=M(R)$ in $(6)$, where $R$ is the radius of the star. We here remark that this model does not admit the zero radius limit with positive $M(R)$ fixed, since the stress tensor of the Schwarzschild black hole given by Eq. (16) is not the form of the perfect fluid, (27). This shows that there needs to be a certain dynamical process for a star described by (26) and (27) to collapse into the Schwarzschild black hole. We shall confirm this explicitly in the analysis for constant density. 


\subsection{Total energy with an equation of state}

We define the total energy of this system by the conserved charge corresponding to the Killing vector $\xi^{\mu}=-\delta_{0}^{\mu}$. ${ }^{\mathrm{b}}$ Then it can be computed as

$$
E=V_{d-2} \int_{0}^{R} d r \sqrt{f(r) h(r)} r^{d-2} \rho(r) .
$$

Employing (28) and (31), we find

$$
\begin{aligned}
E & =\frac{(d-2) V_{d-2}}{8 \pi}(M(R)+\Delta M), \\
\Delta M & =-\frac{8 \pi G_{N}}{d-2} \int_{0}^{R} d r \sqrt{f(r) h(r)^{3}} r M(r)\{\rho(r)+P(r)\} .
\end{aligned}
$$

The first leading term gives the gravitational mass of the star also called as the Misner-Sharp mass, $\frac{30}{2}$ which is directly related to an actual observable quantity appearing in the metric around the spacial infinity, while the second term is a deviation from the gravitational mass, which corresponds to the gravitational binding energy as we shall see.

In order to investigate the deviation term in more detail, ${ }^{c}$ let us impose the matter consisting of the star to satisfy an equation of state

$$
P(r)=w \rho(r) \text {. }
$$

Plugging this into (28) leads to $f=\rho^{-\frac{2 w}{(1+w)}}$, where we fixed the integration constant to satisfy $f \rightarrow 1$ in the Newtonian limit $w \rightarrow 0 .{ }_{-}^{\mathrm{d}}$ Then the deviation term becomes

$$
\Delta M=-\frac{8 \pi G_{N}(1+w)}{d-2} \int_{0}^{R} d r r \rho^{\frac{1}{(1+w)}} \frac{1}{\left(\frac{-2 \Lambda r^{2}}{(d-1)(d-2)}+1-\frac{2 G_{N} M(r)}{r^{d-3}}\right)^{3 / 2}} M(r) .
$$

Let us expand this in terms of the cosmological constant and the Newton constant. The leading and next-to-leading terms are given by

$$
\begin{aligned}
& \Delta M_{1}=-\frac{8 \pi G_{N}(1+w)}{d-2} \int_{0}^{R} d r r \rho^{\frac{1}{(1+w)}} M(r), \\
& \Delta M_{2}=-\frac{24 \pi G_{N}(1+w)}{d-2} \int_{0}^{R} d r \rho^{\frac{1}{(1+w)}}\left(\frac{\Lambda r^{3}}{(d-1)(d-2)}+\frac{G_{N} M(r)}{r^{d-4}}\right) M(r) .
\end{aligned}
$$

\footnotetext{
b There is a traditional argument for the definition of the total energy of a compact star and its interpretation, which is different from our result presented below. Just for clarity and to avoid confusion, we present our argument in main text and make the comparison to a traditional result in App. A.

${ }^{c}$ We would like to thank Profs. Shibata, Yamaguchi and Mukohyama to motivate us to study the deviation from the Misner-Sharp mass.

$\mathrm{d}$ This integration constant can be also absorbed by rescaling the time variable.
} 
Here $\Delta M_{1}$ represents the leading order of the gravitational self-interaction energy of the matter inside the star obeying the equation of state (35). In particular, at four dimensions in the Newtonian limit $w \rightarrow 0$, this can be rewritten as

$$
\Delta M_{1}=-\frac{G_{N}}{2} \int d^{3} x d^{3} y \frac{\rho(\mathbf{x}) \rho(\mathbf{y})}{|\mathbf{x}-\mathbf{y}|},
$$

which is nothing but Newtonian gravitational energy inside the star including the symmetric factor $1 / 2$.

\subsection{Estimation with constant density}

In order to estimate the size of the correction $\Delta M$, let us consider a case of constant density $\rho(r)=\rho_{0}$ and vanishing cosmological constant. In this case, we can compute $M(r)$ given in $(31)$ as $M(r)=\frac{8 \pi r^{d-1} \rho_{0}}{(d-2)(d-1)}$, which leads to $h(r)^{-1}=1-\frac{r^{2}}{r_{0}^{2}}$ with $r_{0}=$ $\sqrt{\frac{(d-2)(d-1)}{16 \pi G_{N} \rho_{0}}}$. The TOV equation with the boundary condition is easily solved as

$$
P(r)=\frac{\rho_{0}(d-3)\left(\sqrt{r_{0}^{2}-r^{2}}-\sqrt{r_{0}^{2}-R^{2}}\right)}{-(d-3) \sqrt{r_{0}^{2}-r^{2}}+(d-1) \sqrt{r_{0}^{2}-R^{2}}} .
$$

For a stable star the pressure has to be finite for all $r$. This leads to an inequality

$$
R<\frac{2 \sqrt{d-2}}{d-1} r_{0}=: R_{*}
$$

$\Delta M$ can be written as

$$
\Delta M=-\frac{(d-1) \sqrt{r_{0}^{2}-R^{2}}}{4 G_{N} r_{0}^{2}} \int_{0}^{R} d r \frac{r^{d}}{\left(r_{0}^{2}-r^{2}\right)^{3 / 2}} .
$$

At four dimensions, this is computed as

$$
F(R):=\frac{\Delta M}{M(R)}=-\frac{3}{8 G_{N} M(R)}\left(-\frac{R^{3}}{r_{0}^{2}}+3 R-3 \sqrt{r_{0}^{2}-R^{2}} \arctan \left(\frac{R}{\sqrt{r_{0}^{2}-R^{2}}}\right)\right),
$$

where $F(R)$ turns out to be a monotonically decreasing function from $F(0)=0$ to $F\left(R_{*}\right) \simeq-0.68$. Therefore the total energy $E$ can be about one third of $M(R)$ so that the gravitational binding energy could be considerably large. In particular the total energy of a static compact star becomes smaller than the gravitational mass observed at spacial infinity due to the gravitational self-interaction.

e This inequality leads to the lower bound for the radius of the star $R_{\min }$ as $R^{d-3} \geq$ $\frac{(d-1)^{2}}{2(d-2)} G_{N} M_{0}=R_{\text {min }}^{d-3}$ for keeping $M(R)=M_{0}$ independent on $R$. This is consistent with the previous argument that this system does not admit the zero size limit fixing a positive $M(R)=M_{0}$. 


\section{Discussion}

We have proposed a general definition of a conserved charge from any covariantly conserved current, which requires no specific asymptotic behaviors/approximations for the metric, or no subtraction of boundary contributions, as long as a Killing vector exists. Our definition has reproduced the mass, electric charge and angular momentum of black holes known in the literature. Since the presented formula requires the matter energy-momentum tensor to define the mass, it is clear that black holes inevitably have nonzero matter energy-momentum tensor at singularity (see also Ref. 31 in the case of higher derivative gravity). One of the important consequences in this paper is that any black hole is not a vacuum solution in this treatment. ${ }^{f}$ This is similar to an electron in electrodynamics: its electric charge distribution is described by a delta function at its position and it is not regarded as a vacuum solution. We have also demonstrated that the total energy of any spherically symmetric compact star defined as the conserved charge cannot be written as a surface term alone, and it contains the gravitational binding energy in addition to the gravitational mass observed at the asymptotic spatial infinity.

Our definition of charges is formulated as a generalization of ones used in the flat space-time to be available in a general curved space-time. This is achieved to enjoy general covariance manifestly. Therefore our definition has clear physical meaning and is of a generic use with precision compared to other definitions. We discuss a difference of a couple of other approaches more in App. A.

In this paper we focused on a few well-known black holes such as the Schwarzschild and BTZ ones to compute their charges. In these simple cases the matter stress tensor is described by a delta function singularity at the origin. It would be interesting to study a more intricate black hole whose singularity is not pointlike any more. In such a case we expect that matter energy-momentum tensor is still described by a certain integrable distribution and our definition of charges is valid and useful also as numerical evaluation. (Distributional expressions for the Kerr black hole can be found in Ref. 23.)

Our proposal is quite generic, so we expect plenty of applications in future. As such a potential application, we consider a more general case where any Killing vectors do not exist. We can still consider a charge or a generator associated with a general vector field $\xi^{\mu}$ as

$$
Q[\xi](t)=\int_{M_{t}} d^{d-1} \mathbf{x} \sqrt{|g|} T^{0}{ }_{\nu} \xi^{\nu}
$$

Using the similar argument before, we obtain

$$
\frac{d Q[\xi]}{d t}=\int_{M_{t}} d^{d-1} \mathbf{x} \sqrt{|g|} \rho(x), \quad \rho(x):=\frac{\left(G^{\mu \nu}+\Lambda g^{\mu \nu}\right)}{16 \pi G_{N}}\left(\nabla_{\mu} \xi_{\nu}+\nabla_{\nu} \xi_{\mu}\right),
$$

\footnotetext{
${ }^{\mathrm{f}}$ Traditionally black holes including the ones dealt with in this paper may have been regarded as vacuum solutions in space-time with their singularity deficient. The method presented in this paper does not return inconsistent results with the traditional treatment, but is applicable to any gravitational object on a general curved space-time such as a black hole and a compact star.
} 
where $\rho=0$ if $\xi$ is a Killing vector. A change of the charge $Q[\xi]$ can be calculated by the volume integral of $\rho$, expressed in terms of the gravitational field through the Einstein equation with the manifest covariance being kept. Equation (45) may give a hint for a general conservation equation in general relativity. Note that this does not require any pseudo-tensor. This argument will hold not only for a Lorentzian manifold but also for a more general one. We will return to this interesting problem in future studies.

\section{Acknowledgments}

We would like to thank Profs. S. Mukoyama, J. H. Park, M. Sasaki, M. Shibata, T. Shiromizu, S. Sugimoto and S. Yamaguchi for useful discussions and valuable comments. This work is supported in part by the Grant-in-Aid of the Japanese Ministry of Education, Sciences and Technology, Sports and Culture (MEXT) for Scientific Research (Nos. JP16H03978, JP18K03620, JP18H05236 and JP19K03847). S. Aoki is also supported in part by a priority issue (Elucidation of the fundamental laws and evolution of the universe) to be tackled by using Post "K" Computer, and by Joint Institute for Computational Fundamental Science (JICFuS). T. Onogi would like to thank YITP for their kind hospitality during his stay for the sabbatical leave from his home institute.

\section{Appendix A. Comparison with other Definitions}

\section{A.1. The Komar integral}

In this appendix, we compare our results with those obtained by the Komar integral,,$\frac{16}{}$ defined as

$$
\begin{aligned}
E_{\text {Komar }}(\xi) & =\frac{c}{16 \pi G_{N}} \int_{M_{t}} d^{d-1} \mathbf{x} \sqrt{|g|} \nabla_{\mu} \nabla^{[0} \xi^{\mu]} \\
& =\frac{c}{16 \pi G_{N}} \int_{\partial M_{t}}\left[d^{d-2} \mathbf{x}\right]_{\mu} \sqrt{|g|} \nabla^{[0} \xi^{\mu]}
\end{aligned}
$$

where $c$ is some constant, $\partial M_{t}$ is the boundary of $M_{t}$ and $\left[d^{d-2} \mathbf{x}\right]_{\mu}$ is its (hyper-) surface element normal to the $\mu$ direction. The second expression corresponds to the quasi-local definition.

While our result (13) for the energy of the Schwarzschild black hole is independent of $\Lambda$, the Komar energy (A.1) with the Killing vector diverges for $\Lambda \neq 0$. For the vanishing cosmological constant, we obtain

$$
E_{\text {Komar }}(\xi)=\frac{c V_{d-2}(d-3) M}{8 \pi},
$$

which agrees with Eq. (13) at $d>3$ if we take $c=(d-2) /(d-3)$. 
The divergence in the energy of the charged black hole also appears in the Komar energy if it is evaluated by the volume integral in the first expression of Eq. (A.1), while such a divergent term vanishes in the Komar energy evaluated by the surface integral in the second expression of Eq. (A.1). This explicitly demonstrates that the Stokes' theorem does not hold if the volume integral has divergence, which cannot be detected by the surface integral, warning that some care is needed to make a conclusion for conserved quantities by the quasi-local expression.

The Komar energy for the BTZ black hole diverges due to the nonzero cosmological constant, while the Komar angular momentum agrees with our result, $P_{\phi}=\frac{J}{8}$, with $c=1$ for a constant.

In the case of a compact star energy, the Komar energy at $\Lambda=0$ (otherwise it diverges) becomes a pure surface term contribution as

$$
E_{\mathrm{Komar}}=\frac{c(d-3) V_{d-2}}{8 \pi} M(R) .
$$

Let us briefly mention some other evaluations. At $\Lambda=0$, Ref. 9 gives the mass of both neutral and charged black holes as $E_{\mathrm{BY}}=E_{\text {ours }}$ at $d=4$ while the mass of the compact star as $E_{\mathrm{BY}}(R)=M(R)$ at $d=4$. In the case of the AdS space with $\Lambda<0$, Ref. 12 gives

$$
E=\frac{M}{4}, \quad P_{\phi}=\frac{J}{8}
$$

for the BTZ blackhole, $E_{\mathrm{AdS}_{4}}=E_{\text {ours }}$ at $d=4$ and $E_{\mathrm{AdS}_{5}}=E_{\text {ours }}+\frac{3 \pi \ell^{2}}{32 G_{N}}$ at $d=5$, where $\ell$ is the radius of $\mathrm{AdS}_{5}$ related to the cosmological constant as $\Lambda=-\frac{6}{\ell^{2}}$. The deviation from our result appears after the divergence due to the cosmological constant term is canceled by adding possible quasi-local counterterms. We also mention that Ref. 13 gives the same result as ours for the neutral black hole based on the surface integral using the "effective stress-energy tensor."

We summarize the above comparisons in Table A.1.

Table A.1. A summary of comparisons, where $E_{\text {ours }}=\frac{(d-2) V_{d-2} M}{8 \pi}, E_{\text {ours }}(R)=\frac{(d-2) V_{d-2} M(R)}{8 \pi}$, $E_{\mathrm{Komar}}=\frac{c(d-3) V_{d-2} M}{8 \pi}, E_{\mathrm{Komar}}(R)=\frac{c(d-3) V_{d-2} M(R)}{8 \pi}, E_{\mathrm{BY}}=M, E_{\mathrm{BY}}(R)=M(R)$, and $\delta E=\frac{3 \pi \ell^{2}}{32 G_{N}}$. In the case of the Komar energy, we set $\Lambda=0$ except for the BTZ black hole, otherwise it diverges. The Komar energy for the charged black hole is evaluated by volume integral and by surface integral, where the latter is shown in the parenthesis.

\begin{tabular}{l|c|c|cc}
\hline & Neutral BH & Charged BH & BTZ BH & Compact star \\
\hline Ours & $E_{\text {ours }}$ & $E_{\text {ours }}+\infty$ & $E=\frac{M}{4}, P_{\phi}=\frac{J}{8}$ & $E_{\text {ours }}(R)+\Delta M$ \\
Komar (quasi-local) & $E_{\text {Komar }}$ & $E_{\text {Komar }}+\infty\left(E_{\text {Komar }}\right)$ & $E=\infty, P_{\phi}=\frac{c J}{8}$ & $E_{\text {Komar }}(R)$ \\
Ref. 9 $(\Lambda=0, d=4)$ & $E_{\mathrm{BY}}$ & $E_{\mathrm{BY}}+0$ & - & $E_{\mathrm{BY}}(R)$ \\
\hline Ref. $12(\Lambda<0, q=0)$ & $E_{\mathrm{AdS}_{4}}=E_{\text {ours }}, E_{\mathrm{AdS}_{5}}=E_{\text {ours }}+\delta E$ & $E=\frac{M}{4}, P_{\phi}=\frac{J}{8}$ & - \\
Ref. $\overline{13}(\Lambda<0, q=0)$ & \multicolumn{2}{|c|}{$E_{\mathrm{AdS}_{4}}=E_{\mathrm{ours}}, E_{\mathrm{AdS}_{5}}=E_{\mathrm{ours}}$} & - & - \\
\hline
\end{tabular}




\section{A.2. The Misner-Sharp mass}

In this appendix, we compare our result with others on the total energy of a static compact star at four dimensions and zero cosmological constant.

Our final result is given by (32). (This expression is also written in appendix in Ref. 32.) On the other hand, a traditional argument written in standard textbooks ${ }^{33-37}$ is that the total energy of a static compact star is given only by the gravitational mass known as the Misner-Sharp mass, ${ }^{30}$ denoted by $M(R)$ in this paper, and that the gravitational binding energy is included in the gravitational mass even though $M(R)$ does not have any nontrivial dependence on the metric as seen from its expression (31).

According to the textbooks, to compute the gravitational potential energy, one needs to subtract the sum of the static mass and the internal energy from the Misner-Sharp mass:

$$
\Omega=M(R)-\varepsilon,
$$

where $\varepsilon$ is the sum of the static mass and the internal energy. However two different ways to compute $\varepsilon$ are described in the textbooks. One way is to use the volume form in the Cauchy surface, ${ }^{34-36}$ which can be written in our convention as

$$
\varepsilon^{(1)}=\int_{0}^{R} \rho \sqrt{h \tilde{g}} d^{3} x .
$$

The other way is to use the volume form in the total space-time: ${ }^{33,37}$

$$
\varepsilon^{(2)}=\int_{0}^{R} \rho \sqrt{|g|} d^{3} x .
$$

Note $\varepsilon^{(2)}=E$ (see Eq. (32)). What is unsatisfactory in the first method is that it does not respect the general covariance any more. An unsatisfactory point in the second method is that the resulting gravitational potential energy, $\Omega^{(2)}=M(R)-\varepsilon^{(2)}$, becomes positive when the density is constant, because $\Omega^{(2)}=-\Delta M$, where $\Delta M$ is negative for a constant density as shown in Subsec. 4.3. For a generic nonconstant density, $\Omega^{(2)}$ is positive while $\Omega^{(1)}$ is negative in the Newtonian limit. ${ }^{34-36}$ In addition what is unclear in common to both methods is to argue that the Misner-Sharp mass, which is independent of the nontrivial metric, contains gravitational binding energy. The result presented in this paper has no such an unreasonable point.

It would be important to scrutinize these results including us from a different perspective such as the tidal force, or the nonremovable gravitational force of any extended object.

\section{References}

1. A. Einstein, Ann. Phys. Ser. 4 49, 769 (1916).

2. E. Noether, Nachrichten von der Gesellschaft der Wissenschaften zu Göttingen, Mathematisch-Physikalische Klasse (1918), pp. 235-257. 
3. C. W. Misner, K. S. Thorne and J. A. Wheeler, Gravitation (Freeman, San Francisco, 1973), Sec. 20.

4. R. Geroch, Ann. N.Y. Acad. Sci. 224, 108 (1973).

5. L. D. Landau and E. M. Lifshitz, The Classical Theory of Fields, 4th edn. (Pergamon Press, 1975), Sec. 96.

6. R. L. Arnowitt, S. Deser and C. W. Misner, Gravitation: An Introduction to Current Research, ed. L. Witten (Wiley, New York, 1962).

7. R. L. Arnowitt, S. Deser and C. W. Misner, Gen. Relativ. Gravit. 40, 1997 (2008), doi:10.1007/s10714-008-0661-1.

8. H. Bondi, M. van der Burg and A. Metzner, Proc. R. Soc. London A 269, 21 (1962), doi:10.1098/rspa.1962.0161.

9. J. Brown and J. W. York Jr., Phys. Rev. D 47, 1407 (1993), doi:10.1103/PhysRevD. 47.1407, arXiv:gr-qc/9209012.

10. S. Hawking and G. T. Horowitz, Class. Quantum Grav. 13, 1487 (1996), doi:10.1088/ 0264-9381/13/6/017, arXiv:gr-qc/9501014.

11. G. T. Horowitz and R. C. Myers, Phys. Rev. D 59, 026005 (1998), doi:10.1103/ PhysRevD.59.026005, arXiv:hep-th/9808079.

12. V. Balasubramanian and P. Kraus, Commun. Math. Phys. 208, 413 (1999), doi:10. 1007/s002200050764, arXiv:hep-th/9902121.

13. A. Ashtekar and S. Das, Class. Quantum Grav. 17, L17 (2000), doi:10.1088/ 0264-9381/17/2/101, arXiv:hep-th/9911230.

14. S. Aoki, T. Onogi and S. Yokoyama, arXiv:2004.03779 [hep-th].

15. V. Fock, Theory of Space, Time, and Gravitation (Pergamon Press, New York, 1959).

16. A. Komar, Phys. Rev. 127, 1411 (1962), doi:10.1103/PhysRev.127.1411.

17. L. Abbott and S. Deser, Nucl. Phys. B 195, 76 (1982), doi:10.1016/0550-3213(82) 90049-9.

18. P. Townsend, arXiv:gr-qc/9707012.

19. A. Trautman, King's College lecture notes on general relativity, mimeographed notes, May-June 1958 (unpublished).

20. A. Trautman, Gen. Relativ. Gravit. 34, 721 (2002), doi:10.1023/A:1015939926662.

21. A. Chamblin, R. Emparan, C. V. Johnson and R. C. Myers, Phys. Rev. D 60, 064018 (1999), doi:10.1103/PhysRevD.60.064018, arXiv:hep-th/9902170.

22. H. Balasin and H. Nachbagauer, Class. Quantum Grav. 10, 2271 (1993), doi:10.1088/ 0264-9381/10/11/010, arXiv:gr-qc/9305009.

23. H. Balasin and H. Nachbagauer, Class. Quantum Grav. 11, 1453 (1994), doi:10.1088/ 0264-9381/11/6/010, arXiv:gr-qc/9312028.

24. A. S. Eddington, Nature 113, 192 (1924).

25. D. Finkelstein, Phys. Rev. 110, 965 (1958), doi:10.1103/PhysRev.110.965.

26. M. Banados, C. Teitelboim and J. Zanelli, Phys. Rev. Lett. 69, 1849 (1992), doi:10. 1103/PhysRevLett.69.1849, arXiv:hep-th/9204099.

27. M. Banados, M. Henneaux, C. Teitelboim and J. Zanelli, Phys. Rev. D 48, 1506 (1993), doi:10.1103/PhysRevD.48.1506, arXiv:gr-qc/9302012.

28. J. Oppenheimer and G. Volkoff, Phys. Rev. 55, 374 (1939), doi:10.1103/PhysRev.55. 374.

29. R. C. Tolman, Phys. Rev. 55, 364 (1939), doi:10.1103/PhysRev.55.364.

30. C. W. Misner and D. H. Sharp, Phys. Rev. 136, B571 (1964), doi:10.1103/PhysRev. 136.B571.

31. L. Buoninfante, A. S. Koshelev, G. Lambiase, J. Marto and A. Mazumdar, J. Cosmol. Astropart. Phys. 06, 014 (2018), doi:10.1088/1475-7516/2018/06/014, arXiv:1804. 08195 [gr-qc]]. 
32. S. Angus, K. Cho and J. H. Park, Eur. Phys. J. C 78, 500 (2018), doi:10.1140/epjc/ s10052-018-5982-y, arXiv:1804.00964 [hep-th].

33. S. Weinberg, Gravitation and Cosmology: Principles and Applications of the General Theory of Relativity (John Wiley and Sons, 1972).

34. C. W. Misner, K. S. Thorne and J. A. Wheeler, Gravitation (Freeman, San Francisco, 1973), Sec. 23.

35. S. W. Hawking and G. F. R. Ellis, The Large Scale Structure of Space-Time (Cambridge University Press, 1973), doi:10.1017/CBO9780511524646.

36. R. M. Wald, General Relativity (University Chicago Press, 1984), doi:10.7208/ chicago/9780226870373.001.0001.

37. B. F. Schutz, A First Course in General Relativity, 2nd edn. (Cambridge University Press, 2009). 